\title{
Measuring carrier density in parallel conduction layers of quantum Hall systems
}

\author{
M. Grayson, F. Fischer \\ Walter Schottky Institut, Technische Universität München, \\ D-85748 Garching, Germany
}

(Dated: 17 Nov 2004)

(Rsion 6)

\begin{abstract}
An experimental analysis for two parallel conducting layers determines the full resistivity tensor of the parallel layer, at magnetic fields where the other layer is in the quantum Hall regime. In heterostructures which exhibit parallel conduction in the modulation-doped layer, this analysis quantitatively determines the charge density in the doping layer and can be used to estimate the mobility. To illustrate one application, experimental data show magnetic freeze-out of parallel conduction in a modulation doped heterojunction. As another example, the carrier density of a minimally populated second subband in a two-subband quantum well is determined. A simple formula is derived that can estimate the carrier density in a highly resistive parallel layer from a single Hall measurement of the total system.
\end{abstract}

Various high-mobility heterostructures contain parallel conducting layers whose individual conductance parameters need to be characterized. Such systems include double-quantum well systems and quantum wells with a second occupied subband. Another system of practical interest is a high-mobility two-dimensional electron system (2DES) with a parallel conducting modulationdopant layer. In all cases, the well-known signature of parallel conduction is that the minima in the longitudinal resistance $R_{x x}$ do not go to zero in the quantum Hall limit. One standard method for separately characterizing the layer densities is the Shubnikov-de Haas $(\mathrm{SdH})$ method, which requires that both systems show oscillations in $R_{x x}$ in the same magnetic field range $B$. These oscillations are then Fourier transformed against $1 / B$ to give the densities of both systems 1 . However, this method cannot be used if one of the systems has sufficiently low mobility that $\mathrm{SdH}$ oscillations are indiscernable, and this method gives no separable measurement of $R_{x x}$ for each layer. Other methods have been introduced which can determine resistivities in non-quantized parallel channels 2, 3, [4], but to date an explicit treatment of the quantized case is lacking. In this paper, we present a new analysis of magnetotransport data which determines both components of the resistivity tensor $\rho_{x x}^{\|}$and $\rho_{x y}^{\|}$in one layer, at values of the $B$ field where the other layer is in the quantized Hall regime. The density of carriers in the parallel layer then follows directly $n^{\|}=B / \rho_{x y}^{\|} e$, with $e$ the electron charge.

We wish to first find an expression for the resistivity tensor of the combined quantum Hall and parallel conducting layers since resistivity is the experimentally measured quantity. We define separate resistivity tensors for the quantum Hall and parallel conduction layers as:

$$
\rho^{\mathbf{Q}}=\left(\begin{array}{cc}
0 & \frac{h}{\nu e^{2}} \\
-\frac{h}{\nu e^{2}} & 0
\end{array}\right), \quad \rho^{\|}=\left(\begin{array}{cc}
\rho_{x x} & \rho_{x y}^{\|} \\
-\rho_{x y}^{\|} & \rho_{x x}^{\|}
\end{array}\right)
$$

Assuming that the in-plane electric fields seen by the two layers are equal, the corresponding conductivities for each layer,

$$
\sigma^{\mathbf{Q}}=\left(\begin{array}{cc}
0 & -\frac{\nu e^{2}}{h} \\
\frac{\nu e^{2}}{h} & 0
\end{array}\right), \quad \sigma^{\|}=\frac{1}{|\rho \||}\left(\begin{array}{cc}
\rho_{x x}^{\|} & -\rho_{x y}^{\|} \\
\rho_{x y}^{\|} & \rho_{x x}^{\|}
\end{array}\right)
$$

can be summed to yield the sheet conductivity of the combined system

$$
\sigma^{\text {tot }}=\sigma^{\mathbf{Q}}+\sigma^{\|}=\frac{\mathbf{1}}{\left|\rho^{\|}\right|}\left(\begin{array}{cc}
\rho_{x x}^{\|} & -\rho_{x y}^{\|}-\left|\rho^{\|}\right| \frac{\nu e^{2}}{h} \\
\rho_{x y}^{\|}+\left|\rho^{\|}\right| \frac{\nu e^{2}}{h} & \rho_{x x}^{\|}
\end{array}\right)
$$

where $\left|\rho^{\|}\right|=\operatorname{det}\left(\rho^{\|}\right)$. Inverting $\sigma^{\text {tot }}$ gives the total resistivity tensor, $\rho^{\text {tot }}=\left(\sigma^{\text {tot }}\right)^{-1}$ whose components are directly measured in experiment:

$$
\rho_{x x}^{t o t}=\frac{\left|\rho^{\|}\right| \rho_{\mathbf{x x}}^{\|}}{\rho_{x x}^{\|{ }^{\prime \prime}}+\left(\left|\rho^{\|}\right| \frac{\nu \mathbf{e}^{2}}{\mathrm{~h}}+\rho_{\mathbf{x y}}\right)^{2}}, \quad \rho_{x y}^{t o t}=\frac{\left|\rho^{\|\|}\right|\left(\left|\rho^{\|}\right| \frac{\nu \mathbf{e}^{2}}{\mathrm{~h}}+\rho_{\mathbf{x y}}^{\|}\right)}{\rho_{x x}^{\|{ }^{2}}+\left(\left|\rho^{\|}\right| \frac{\nu^{2}}{\mathrm{~h}}+\rho_{\mathbf{x y}}\right)^{2}}
$$

Just as in single-layer Hall measurements of where expressions for the longitudinal and transverse resistance are solved for the two unknowns, density and Hall mobility, the above equations can be solved for the only two unknowns in this problem, namely $\rho_{x x}^{\|}$and $\rho_{x y}^{\|}$, the components of the resistivity tensor in the parallel layer. We will demonstrate this analysis for data measured in the general Van der Pauw geometry, although the same analysis can be adapted to Hall bar and Corbino geometries. All 4-point resistances were measured in an Oxford ${ }^{3} \mathrm{He}$ cryostat using standard lock-in techniques with a high input impedance pre-amplifier $\left(R_{i n} \sim 100 \mathrm{G} \Omega\right)$ to avoid measurement errors which might be mistaken for parallel conduction [5].

The first sample of interest is a heterojunction quantum well with parallel conduction in the modulation doping layer. We start experimentally by measuring the longitudinal resistance of the sample at finite B-field in two 
complementary geometries $R_{x x}^{A}$ and $R_{x x}^{B}$ (see Fig. 2), and determine the total $B$-dependent sheet resistance $\rho_{x x}^{t o t}(B)$ according to the Van der Pauw equations [ 6 ]:

$$
\begin{aligned}
\rho_{x x}^{t o t} & =\frac{\pi}{\ln (2)} f(x) \frac{R_{x x}^{A}+R_{x x}^{B}}{2} \\
f(x) & =1-\frac{\ln 2}{2}\left(\frac{x-1}{x+1}\right)^{2}-\left[\frac{(\ln 2)^{2}}{4}-\frac{(\ln 2)^{3}}{12}\right]\left(\frac{x-1}{x+1}\right)^{4}
\end{aligned}
$$

Here $x=R_{x x}^{A} / R_{x x}^{B}$ for $R_{x x}^{A}>R_{x x}^{B}$, and $f(x) \leq 1$ is a weak function of $x$ of order unity. The Hall resistance $\rho_{x y}^{t o t}=R_{x y}$ can be measured directly (Fig. 2).

We now solve Eq. 4 for $\rho_{x x}^{\|}$and $\rho_{x y}^{\|}$in terms of the measured $\rho_{x x}^{\text {tot }}$ and $\rho_{x y}^{\text {tot }}$. The total resistivity tensor $\rho^{\text {tot }}$ inverted to give $\sigma^{\text {tot }}=\left(\rho^{\text {tot }}\right)^{-1}$, and since $\sigma^{\text {tot }}=\sigma^{\mathbf{Q}}+$ $\sigma^{\|}$,

$$
\sigma_{x x}^{t o t}=\sigma_{x x}^{\|}=\frac{\rho_{x x}^{t o t}}{\left|\rho^{t o t}\right|}, \quad \sigma_{x y}^{t o t}=\sigma_{x y}^{\|}+\frac{\nu e^{2}}{h}=\frac{\rho_{x y}^{t o t}}{\left|\rho^{t o t}\right|}
$$

Knowing the filling factor index $\nu$ of a given quantum Hall minimum, we solve for $\sigma_{x x}^{\|}=\sigma_{x x}^{t o t}$ and $\sigma_{x y}^{\|}=\sigma_{x y}^{t o t}-$ $\frac{\nu e^{2}}{h}$. Inverting $\sigma^{\|}$to find $\rho^{\|}=\left(\sigma^{\|}\right)^{-\mathbf{1}}$ yields:

$$
\begin{aligned}
& \rho_{x x}^{\|}=\frac{\sigma_{x x}^{\|}}{\left|\sigma^{\|}\right|}=\frac{\left|\rho^{\mathbf{t o t}}\right| \rho_{\mathbf{x x}}^{\mathbf{t o t}}}{\left(\rho_{x x}^{t o t}\right)^{2}+\left(\rho_{x y}^{t o t}-\left|\rho^{\mathbf{t o t}}\right| \frac{\nu \mathbf{e}^{2}}{\mathbf{h}}\right)^{2}} \\
& \rho_{x y}^{\|}=\frac{\sigma_{x y}^{\|}}{\left|\sigma^{\|}\right|}=\frac{\left|\rho^{\mathbf{t o t}}\right|\left(\rho_{\mathbf{x y}}^{\mathbf{t o t}}-\left|\rho^{\mathbf{t o t}}\right| \frac{\nu \mathbf{e}^{\mathbf{2}}}{\mathbf{h}}\right)}{\left(\rho_{x x}^{t o t}\right)^{2}+\left(\rho_{x y}^{t o t}-\left|\rho^{\mathbf{t o t}}\right| \frac{\nu \mathbf{e}^{\mathbf{2}}}{\mathbf{h}}\right)^{2}}
\end{aligned}
$$

which is nothing other than Eq. (44) solved for $\rho_{x x}^{\|}$and $\rho_{x y}^{\|}$. The result is plotted in Fig. 目for values of $B$ where the 2DES Hall resistance is quantized.

We can estimate the carrier density in the parallel layer with either of two methods. First, the slope of $\rho_{x y}^{\|\|}$(both within each quantum Hall effect domain and across all filling factors) indicates a density of $n^{\|}=$ $\frac{B}{e \rho_{x y}^{\|}}=0.30 \times 10^{11} \mathrm{~cm}^{-2}$. Secondly, as a useful backof-the-envelope shorthand, we note that in the limit of $\rho_{x x}^{t o t}<\rho_{x y}^{t o t}$ as in Fig. 3. Eq. (8) can be simplified to,

$$
\rho_{x y}^{\|}=\frac{B}{n^{\|_{e}}} \approx \frac{\rho_{x y}^{t o t}}{1-\rho_{x y}^{t o t} \frac{\nu e^{2}}{h}}
$$

Solving for $n^{\|}$gives

$$
n^{\|} \approx n^{Q}\left(\frac{\frac{h}{\nu e^{2}}}{\rho_{x y}^{t o t}}-1\right)
$$

where $n^{Q}=\frac{\nu e B(\nu)}{h}$ is the density of the QH system, and $B(\nu)$ is the magnetic field value in the center of the $\nu$-th plateau in $\rho_{x y}^{t o t}$. In Fig. 3 the flat lines above each $\rho_{x y}^{t o t}$ plateau show the resistance quantum $\frac{h}{\nu e^{2}}$ demonstrating that the first term in parenthesis in Eq. (10) is always greater than 1. The remarkable utility of Eq. (10) is that the parallel carrier density can be estimated from a single $\rho_{x y}^{\text {tot }}$ measurement. For example in $\rho_{x y}^{\text {tot }}$ from Fig. 3] the position of the $\nu=1$ plateau at $B(\nu=1)=8.07 \mathrm{~T}$ determines $n^{Q}=1.95 \times 10^{11} \mathrm{~cm}^{-2}$ and the plateau resistance of $\Delta \rho_{x y}^{t o t}=22.76 \mathrm{k} \Omega$ gives $n^{\|}=0.26 \times 10^{11} \mathrm{~cm}^{-2}-$ within $15 \%$ of the more exact graphical analysis of Eq. (8) and the resulting Fig. 4]

We can separately characterize the conductance of the parallel delta-doping layer as a result of this analysis. Looking at the two components of the resistivity tensor in Fig. 4 one observes first the sharp rise in $\rho_{x x}^{\|}$resulting from so-called magnetic freeze-out in this highly disordered delta-doping layer, expected in systems with hopping conduction. The total number of mobile carriers in the delta layer, however, stays fixed as confirmed by the constant slope of $\rho_{x y}^{\|}$. We can also make a rough estimate of the mobility of this layer, by extrapolating the parabolic fit shown in the figure to $B=0$. With an intercept resistivity of around $\rho_{x y}^{\|}=7 k \Omega$ and the known density, the $B=0$ mobility of the parallel conduction layer is about $\mu^{\|}=3.0 \times 10^{4} \mathrm{~cm}^{2} /$ Vs. In the temperature dependence shown in Fig. 5 we see $\rho_{x x}^{\|}$increase with decreasing temperature, while $\rho_{x y}^{\|}$remains fixed, indicating a temperature independent density. Both behaviors are consistent with hopping conduction in the delta-layer.

The same analysis can be applied to a two-subband quantum well sample. In Fig. [6] we show the measured $\rho_{x x}^{t o t}$ and $\rho_{x y}^{t o t}$ which are analized to give $\rho_{x x}^{\|}$and $\rho_{x y}^{\|}$as plotted in Fig. 7 The measured parallel carrier density of $n^{\|}=0.11 \times 10^{11} \mathrm{~cm}^{-2}$ is in good agreement with selfconsistent Poisson calculations which predict a second subband occupied to $n_{\text {calc }}^{\|}=0.15 \times 10^{11} \mathrm{~cm}^{-2}$.

We note that the SdH signal from this low density second subband was too weak to give and estimable density using alternate the Fourier transform method. Comparing the resistivity components in Fig. [7 with Fig. [ one sees in $\rho_{x y}^{\|}$that once again the density of carriers is rather constant over the whole $B$-field range. The measured $\rho_{x x}^{\|}$ is of order $50 \Omega$ or less, implying a rather high mobility of over $10 \times 10^{6} \mathrm{~cm}^{2} / \mathrm{Vs}$ for this second subband. Although this value is not quantitatively conclusive, qualitatively it indicates that the low density subband which would normally have a correspondingly low mobility, benefits from the Thomas-Fermi screening of the densely populated subband to screen most of the disorder.

As a final note, we remind the reader that measurement errors arise if the input impedance of the lock-in $R_{\text {in }}$ is too low, and these pathological signals look misleadingly like parallel conduction. With a low $R_{i n}$, finite $R_{x x}$ minima of order $R_{\min }=\left(h / \nu e^{2}\right)^{2} / R_{\text {in }}$ are observed with the astonishing property that the false "parallel" signal disappears upon reversing the current contact polarity. 
Such subtleties in quantum Hall measurement phenomena are discussed in detail elsewhere [5].

In conclusion, we have demonstrated a new analysis for the resistivity of parallel $2 \mathrm{D}$ systems that determines the resistivity tensor of one layer at magnetic fields where the other layer is in the quantum Hall regime. We demonstrate the validity of this analysis for the case of a quantum well with two occupied subbands, as well as for double-quantum wells. We also demonstrate the analysis of carrier density in parallel conducting modulation doped layers. In the latter case, this technique will be particularly useful for crystal growers in characterizing doping efficiency, because it elimates the expensive and time-consuming trial-and-error method currently used for doping calibration of parallel conduction layers.

Acknowledgement - This work was supported in part by the DFG (SFB 348). M.G. thanks the A.v. Humboldt Foundation for support.

(a) Email: mgrayson@alumni.princeton.edu

[1] J. Jo, Y. W. Sen, L. W. Engel, M. B. Santos and M. Shayegan, Phys. Rev. B 46, 9776 (1992).

[2] M. van der Burgt, V. C. Karavolas, F. M. Peeters, J. Singleton, R. J. Nicholas, F. Herlach, J. J. Harris, M. Van Hove and G. Borghs, Phys. Rev. B 52, 12218 (1995).

[3] C. M. Hurd, S. P. McAlister, W. R. McKinnon, B. R. Stewart, D. J. Day, P. Mandeville, and A. J. SpringThorpe, J. Appl. Phys. 63, 4706 (1988).

[4] M. J. Kane, N. Apsley, D. A. Anderson, L. L. Taylor and T. Kerr, J. Phys. C: Solid State Phys. 18, 5629 (1985).

[5] F. Fischer and M. Grayson, submitted to J. Appl. Phys. in tandem with this paper.

[6] L. J. van der Pauw, Philips Res. Rep. 13, 1 (1958). 
a) parallel dopant layer

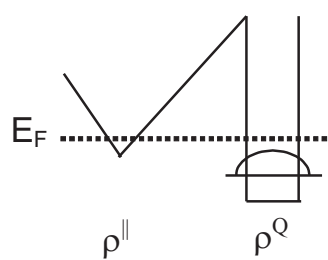

b) parallel quantum well

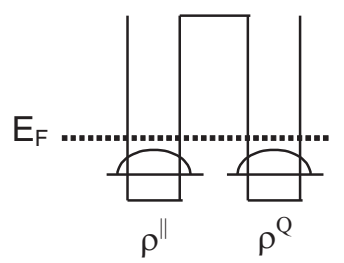

Fig. 1 c) second subband

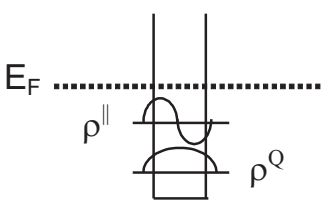

FIG. 1: Conduction band schematic for the three kinds of parallel conduction systems of interest: a) the dopant layer of a modulation-doped heterostructure, b) a double-quantum well system, or c) a quantum well with a second occupied subband. 

a) $R_{x x}^{A}=V_{A} / I$
a) $R_{x x}^{B}=V_{B} / I$
c) $R_{x y}=V_{H} / I$
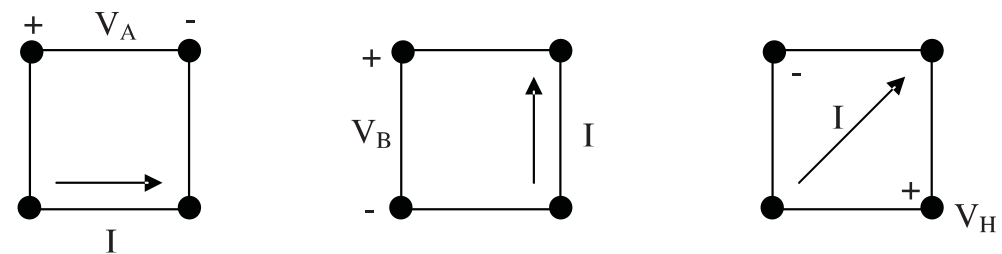

Fig. 2

FIG. 2: The three measurements necessary to completely characterize the resistivity tensor at any magnetic field.

Fig. 3

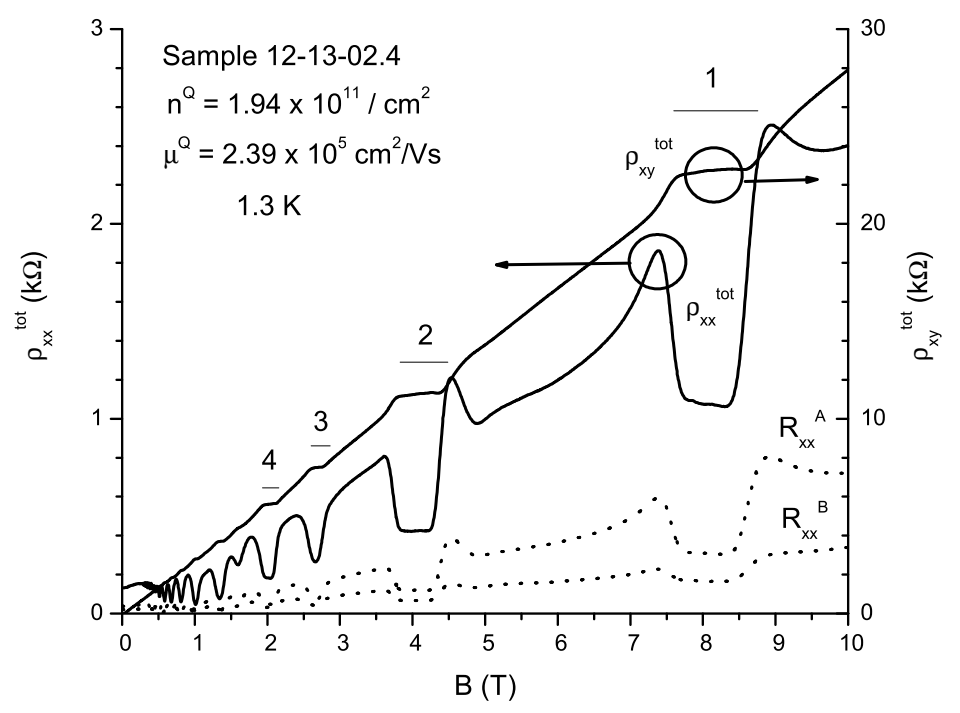

FIG. 3: Plot of measured $\rho_{x x}^{t o t}$, and $\rho_{x y}^{t o t}$ for a sample with parallel conduction in the modulation doped layer. $\rho_{x x}^{t o t}$ is calculated from the Van der Pauw relation in Eq. [5] using $R_{x x}^{A}$ and $R_{x x}^{B}$ as measured in the inset. 
Fig. 4

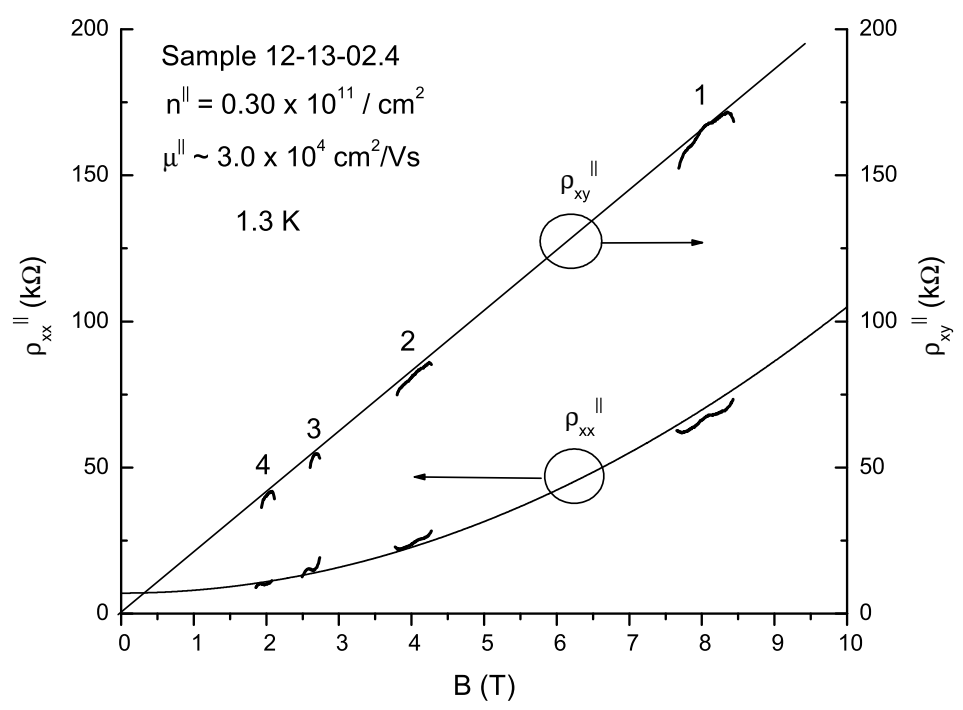

FIG. 4: Plot of $\rho_{x x}^{\|}$and $\rho_{x y}^{\|}$for the parallel conducting modulation doped layer calculated from the data in Fig. 3 using Eqs. 7 and 8 The slope of $\rho_{x y}^{\|}$yields the carrier density in the parallel conductor $n^{\|}=\frac{B}{\rho_{x y}^{\|}} e$.

Fig. 5

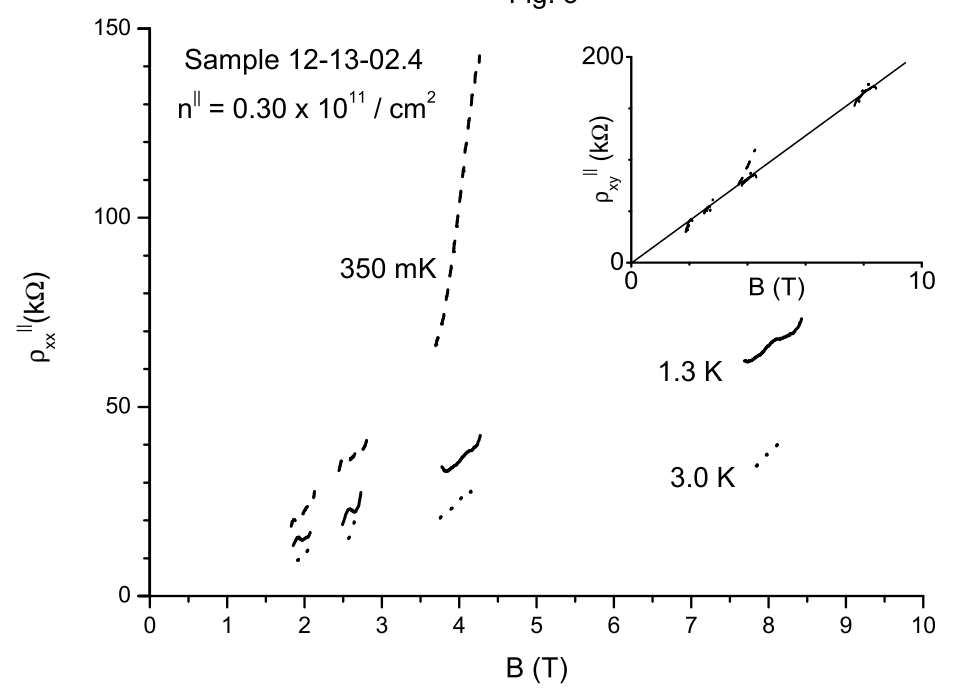

FIG. 5: Plot of $\rho_{x x}^{\|}$for the parallel conducting modulation doped layer at various temperatures. The hopping conduction in this system is strongly temperature and magnetic field dependent. Inset: Plot of $\rho_{x y}^{\|}$for the parallel conducting layer. Note that the density of hopping carriers is independent of $B$ and temperature, except above $4 \mathrm{~T}$ at $350 \mathrm{mK}$ under extreme magnetic freezeout. 
Fig. 6

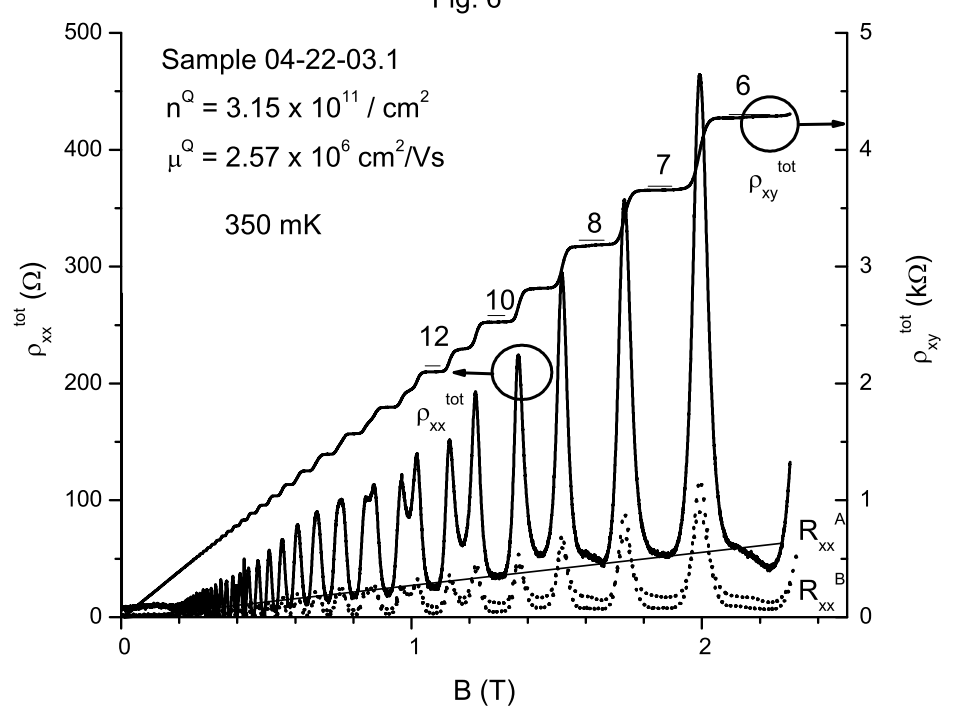

FIG. 6: Plot of $\rho_{x x}^{t o t}, \rho_{x y}^{t o t}, R_{x x}^{A}$ and $R_{x x}^{B}$ for a quantum well with a lightly populated second subband.

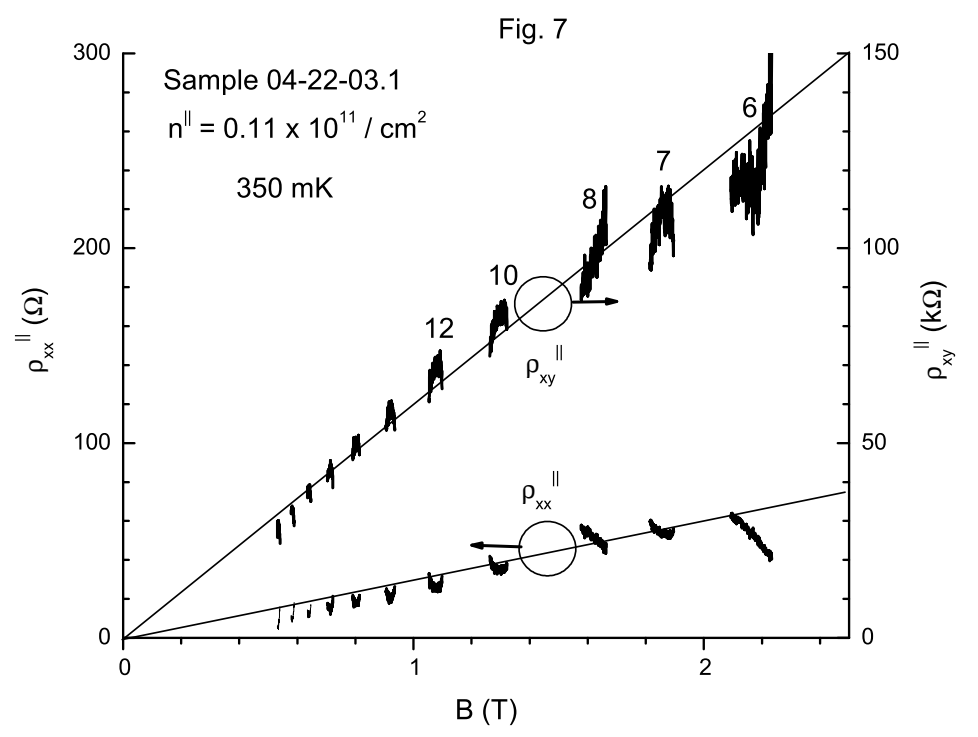

FIG. 7: Plot of calculated $\rho_{x x}^{\|}$and $\rho_{x y}^{\|}$using the data in Fig.

6 for a lightly populated second subband. 